\title{
What Do We Mean by Poor-Grade Aneurysmal Subarachnoid Hemorrhage and What Can We Do?
}

\author{
Julian Bösel ${ }^{1}$ (10)
}

Published online: 7 November 2016

(C) Springer Science+Business Media New York 2016

Aneurysmal subarachnoid hemorrhage (SAH) has been classified poor-grade by the World Federation of Neurosurgery (WFNS) and by the Hunt and Hess grading system as a grade 4 or 5 . In most hospital series, they may account for one quarter of cases [1]. Timing of assessment for prognosis matters and outcome may be better correlated with assessment of WFNS grades after neurocritical stabilization and neurosurgical/neurointerventional procedures [2]. Moreover, the WFNS grading system is broad, based on a Glasgow Coma Scale sum score, and lacks neurosurgical or neurologic details. For reductionists, it is basically coma or not.

Once in the neurocritical care unit (NCCU), the poorgrade SAH patient is mostly understood as a comatose intubated patient with increased intracranial pressure from hydrocephalus and threatened by other potentially serious systemic manifestations of the acute sympathetic surge. Some physicians still assume a bleak course from the onset for these SAH patients, who quickly decline in level of consciousness; but clinical experience has taught us patients may improve quite dramatically if cared for acutely and aggressively.

However, poor-grade SAH in the NCCU requires a detailed set of specific treatment targets-neurologically and medically - that goes beyond prevention of rebleeding. The Neurocritical Care Society has published recommendations focusing on such NCCU measures for SAH [3].

Julian Bösel

julian.boesel@med.uni-heidelberg.de

1 Section of Acute and Critical Care Neurology, Department of Neurology, University Hospital Heidelberg, Im Neuenheimer Feld 400, 69120 Heidelberg, Germany
There is emphasis on initially reducing intracranial pressure including ventriculostomy in acute obstructing hydrocephalus [4], managing potential seizures, managing pulmonary edema, and neurogenic cardiac failure in addition to proceeding with early aneurysm repair. Later, care is directed at avoiding major additional medical complications that would set back achieved gains. It is important to study the outcome of these patients. What has been the impact of neurocritical care over the last decades?

In this issue of Neurocritical Care, de Oliveira Manoel et al. [5] have retrospectively reviewed the clinical outcome of a cohort of 179 poor-grade SAH patients treated at the Trauma and Neurosurgical Intensive Care Unit at St. Michael's Hospital, Toronto, Canada, between 2009 and 2013. They combined their single-center analysis with a thorough systematic review of 23 studies ( $>2700$ patients) on outcome of poor-grade SAH from 1977 to 2014. They included studies reporting outcome at 3 months or more, not excluding patients who died before aneurysmal repair, and thus avoided overestimation of outcome in both directions. Their review yielded an overall mortality rate of $60 \%$ (approximately 30\% dying before and 30\% after aneurysm repair) and favorable outcome in only $28 \%$ of patients. More interestingly, the authors grouped the analysis into decades and showed that while mortality decreased, favorable outcome increased from $13 \%$ in the 1970 s to $30-35 \%$ in the 1990 s, where it has remained since then. This "plateau" in favorable outcome was explained as many more grade-5 SAH patients are admitted to an NCCU today than in the past. In their own cohort, looking at SAH grades WFNS 4 and 5, they found a mortality of 15 and $45 \%$ and favorable outcome in 66 and $28 \%$, respectively. Of note, $57 \%$ of the deceased patients had died after withdrawal of life support. 
In line with two other meta-analyses of outcome studies on poor-grade SAH from 1960 to 1995 [6] and 1995 and 2007 [7], de Oliveira Manoel and colleagues explain the progress from the 1970s to the 1990s as a result of more accurate diagnosis by early computed tomography angiography and digital subtraction angiography, earlier aneurysm treatment to avoid rebleeding, the prophylactic use of nimodipine, and the introduction of coiling. The latter two — both reported around 1990_are still the only single treatments with proof of functional outcome benefit in $\mathrm{SAH}$.

Despite the shortcomings of such a study (retrospective single-center design, review of studies [only 6 of 23 prospective] with heterogeneous outcome assessment, limited potential of scales to detect neurocognitive deficits, and overall daily functioning), the authors have to be commended for their review.

De Oliveira Manoel and colleagues propose the following measures, which may have a critical impact on achieving better outcome in poor-grade SAH: (a) the application of an SAH protocol, (b) early aggressive treatment including external ventricular drain (EVD) placement, (c) the preference of endovascular aneurysm repair and 24/7 access to neurointerventionalists, (d) admission to high-volume centers, and (e) admission to a dedicated NCCU by a multidisciplinary specialized team. This may all sound plausible and, particularly, the latter is indirectly supported by a systematic review by Kramer and Zygun on the effect of dedicated neurocritical care in $>40,000$ mixed brain-injured ICU patients (including $\mathrm{SAH}$ ) reported in almost 20 studies [8]. This overview found that patients receiving care on a specialized NCCU and/or by trained/certified neurocritical care professionals have not only reduced mortality but also reduced poor outcome and are discharged home more often than to longterm institutional care.

"Dedicated neurocritical care" certainly benefits poorgrade SAH patients, but what are the crucial parts of it we should implement and emphasize? How can we make a difference and what factors have an impact on outcome? It is not for lack of trying, but we have mostly seen disappointing results of recent prospective studies showing that statins, magnesium, and some other potential neuroprotectants do not improve outcome in (poor-grade) SAH. A few very interesting randomized trials are currently investigating neurocritical care measures beyond neurosurgery in or including SAH: EARLYDRAIN on early lumbar drainage combined with EVD [9], SETPOINT2 on early tracheostomy [10], NEWTON on slow-release nimodipine via EVD [11], ULTRA on early tranexamic acid [12], and HIMALAIA on induced hypertension [13]. However, there are so many other NCCU aspects to be addressed prospectively: level of sedation, modes of ventilation [14, 15], cardiopulmonary stability [16, 17], monitoring-based circulatory strategies [18], temperature control [19-21], delirium management, the nursing factor and mobilization, policies of do-not-resuscitate orders and withdrawal of care [22, 23], and many other day-to-day care decisions that may substantially impact outcome. Particularly, the poor-grade SAH patient, prone to so many cerebral and systemic complications, may benefit from aggressive surveillance, superb nursing care, and close clinical monitoring. Being there when the patient deteriorates and staying with the patient until the cause is resolved may be just as important as anything else. These aspects urgently deserve research at a higher level of quality, possibly by novel, innovative study designs, possibly by investigating combined therapies.

Entering "subarachnoid hemorrhage" into PubMed as part of the title yields more than 8000 hits. Of these, about 400 are original papers on clinical human studies. Of these, only about 20 relate to intensive care interventions that go beyond aneurysm repair or neuromonitoring. It is hard to understand why there is so little emphasis on management of these poor-grade patients in the literature, and there are good arguments to change that.

\section{Compliance with ethical standard}

Conflicts of interest Dr. Bösel declares no conflict of interest in relation to this article.

\section{References}

1. Wartenberg KE. Critical care of poor-grade subarachnoid hemorrhage. Curr Opin Crit Care. 2011;17(2):85-93.

2. Giraldo EA, Mandrekar JN, Rubin MN, et al. Timing of clinical grade assessment and poor outcome in patients with aneurysmal subarachnoid hemorrhage. J Neurosurg. 2012;117(1):15-9.

3. Diringer MN, Bleck TP, Claude Hemphill J III, et al. Critical care management of patients following aneurysmal subarachnoid hemorrhage: recommendations from the Neurocritical Care Society's Multidisciplinary Consensus Conference. Neurocrit Care. 2011;15(2):211-40.

4. Fried HI, Nathan BR, Rowe AS, et al. The insertion and management of external ventricular drains: an evidence-based consensus statement: a statement for healthcare professionals from the neurocritical care society. Neurocrit Care. 2016;24(1):61-81.

5. de Oliveira Manoel AL, Mansur A, Silva GS, et al. Functional outcome after poor-grade subarachnoid hemorrhage: a singlecenter study and systematic literature review. Neurocrit Care 2016. doi:10.1007/s12028-016-0305-3.

6. Hop JW, Rinkel GJ, Algra A, van Gijn J. Case-fatality rates and functional outcome after subarachnoid hemorrhage: a systematic review. Stroke. 1997;28(3):660-4.

7. Nieuwkamp DJ, Setz LE, Algra A, Linn FH, de Rooij NK, Rinkel GJ. Changes in case fatality of aneurysmal subarachnoid haemorrhage over time, according to age, sex, and region: a metaanalysis. Lancet Neurol. 2009;8(7):635-42. 
8. Kramer AH, Zygun DA. Neurocritical care: why does it make a difference? Curr Opin Crit Care. 2014;20(2):174-81.

9. Bardutzky J, Witsch J, Juttler E, Schwab S, Vajkoczy P, Wolf S. EARLYDRAIN- outcome after early lumbar CSF-drainage in aneurysmal subarachnoid hemorrhage: study protocol for a randomized controlled trial. Trials. 2011;12:203.

10. Schonenberger S, Niesen WD, Fuhrer H, et al. Early tracheostomy in ventilated stroke patients: study protocol of the international multicentre randomized trial SETPOINT2 (Strokerelated Early Tracheostomy vs. Prolonged Orotracheal Intubation in Neurocritical care Trial 2). Int J Stroke 2016;11(3):368-379. doi:10.1177/1747493015616638.

11. Hanggi D, Etminan N, Macdonald RL, et al. NEWTON: Nimodipine Microparticles to Enhance Recovery While Reducing Toxicity After Subarachnoid Hemorrhage. Neurocrit Care. 2015;23(2):274-84.

12. Germans MR, Post R, Coert BA, Rinkel GJ, Vandertop WP, Verbaan D. Ultra-early tranexamic acid after subarachnoid hemorrhage (ULTRA): study protocol for a randomized controlled trial. Trials. 2013;14:143.

13. Gathier CS, van den Bergh WM, Slooter. AJ. HIMALAIA (Hypertension Induction in the Management of AneurysmaL subArachnoid haemorrhage with secondary IschaemiA): a randomized single-blind controlled trial of induced hypertension vs. no induced hypertension in the treatment of delayed cerebral ischemia after subarachnoid hemorrhage. Int $\mathrm{J}$ Stroke. 2014;9(3):375-80.

14. Williamson CA, Sheehan KM, Tipirneni R, et al. The association between spontaneous hyperventilation, delayed cerebral ischemia, and poor neurological outcome in patients with subarachnoid hemorrhage. Neurocrit Care. 2015;23(3):330-8.

15. Westermaier T, Stetter C, Kunze E, et al. Controlled hypercapnia enhances cerebral blood flow and brain tissue oxygenation after aneurysmal subarachnoid hemorrhage: results of a phase 1 study. Neurocrit Care. 2016;25(2):205-14.

16. Schmidt JM, Crimmins M, Lantigua H, et al. Prolonged elevated heart rate is a risk factor for adverse cardiac events and poor outcome after subarachnoid hemorrhage. Neurocrit Care. 2014;20(3):390-8.

17. Chen WL, Chang SH, Chen JH, Tai HC, Chan CM, Wang YC. Heart rate variability predicts neurogenic pulmonary edema in patients with subarachnoid hemorrhage. Neurocrit Care. 2016;25(1):71-8.

18. Kurtz P, Helbok R, Ko SB, et al. Fluid responsiveness and brain tissue oxygen augmentation after subarachnoid hemorrhage. Neurocrit Care. 2014;20(2):247-54.

19. Kramer CL, Pegoli M, Mandrekar J, Lanzino G, Rabinstein AA. Refining the association of fever with functional outcome in aneurysmal subarachnoid hemorrhage. Neurocrit Care 2016. doi:10.1007/s12028-016-0281-7.

20. Seule M, Muroi C, Sikorski C, Hugelshofer M, Winkler K, Keller E. Therapeutic hypothermia reduces middle cerebral artery flow velocity in patients with severe aneurysmal subarachnoid hemorrhage. Neurocrit Care. 2014;20(2):255-62.

21. Kuramatsu JB, Kollmar R, Gerner ST, et al. Is hypothermia helpful in severe subarachnoid hemorrhage? An exploratory study on macro vascular spasm, delayed cerebral infarction and functional outcome after prolonged hypothermia. Cerebrovasc Dis. 2015;40(5-6):228-35.

22. Kowalski RG, Chang TR, Carhuapoma JR, Tamargo RJ, Naval NS. Withdrawal of technological life support following subarachnoid hemorrhage. Neurocrit Care. 2013;19(3):269-75.

23. Choi HA, Fernandez A, Jeon SB, et al. Ethnic disparities in endof-life care after subarachnoid hemorrhage. Neurocrit Care. 2015;22(3):423-8. 